Ca priati (Neapel) setzt die Flektrodiagnostik der progressiven amyotrophischen Lateralsklerose auseinander; besonders durch die Feststellung der Entartungsreaktion sind wir imstande die Diagnose sehr frübzeitig klar zu legen.

Planet (Paris) empfiehlt Faradaysation bei Hydarthrose; besonders in frischen Fällen genügen oft nur wenige Sitzungen zur völligen Heilung. Weil zieht es vor galvanische Ströme, selbst solche von hoher Intensität hierzu zu verwenden; $\mathrm{Oudin}$ und $\mathrm{R}$ onneaux dagegen wenden Hochfrequenzströme an.

Stenbeck (Stockholm) bespricht die Bedeutung fluoreszierender Substanzen bei der Behandlung der Lungentuberkose mit Teslaströmen und findet, dass sie wiederholt sehr überschätzt wurde. Weil warnt vor Anwendung von Hochfequrenzströmen bei florider Tuberkulose.

Laquerrière (Paris) verwendet elektrische Klysmen bei Darmverschluss, starke Faradaysche Ströme bei Diarrhöe, und galvanisiert bei schwerer spastischer Obstipation.

4. In der Sektion für Radiologie berichtet $\mathrm{Bel}$ ot (Paris) über den jetzigen Stand der Technik der Radiotherapie, und über das bisher ausgeübte Messverfahren. Henrard hebt hervor, dass wir heute noch immer nicht im Besitze eines zuverlässigen Messapparates sind.

Guillemin ot (Paris) und Freund (Wien) demonstrieren einige interessante technische Neuigkeiten aus dem Gebiete der Radiologie, deren Zweckmässigkeit von anderen allerdings in Zweifel gezogen wird. Henrard (Brüssel) beschreibt die Extraktionstechnik von Metallkörpern aus der Speiseröhre.

H a u c h a m ps (Brüssel) trägt über Radiodiagnostik vor und verlangt, dass sowohl die Radioskopie als die Radiographie ausschliesslich von Ärzten ausgeübt werde.

Luraschi (Mailand) berichtet über erfolgreiche Tierversuche bei Behandlung der Schilddrüsenhypertrophie mittelst Röntgenstrahlen. W eil (Paris) empfiehlt die Radiotherapie bei Behandlung der Hypertrichose, Desplats (Lille) bei tuberkulöser Adenitis.

In der zweiten allgemeinen Sitzung am 15. August werden in Abwesenheit von Winternitz (Wien) und $\mathrm{Bum}$ (Wien) deren Vorträge über Stellung der Physiotherapie als Unterrichtsgegenstand, sowie über die Kurpfuscherei in den physikalischen Heilmethoden vorgelesen; de $\mathrm{M} \mathrm{unter}$ (Lüttich) spricht über Kurpfuscherei und verlangt, dass alle physikalischen Heilmethoden nur durch Ärzte ausgeübt werden sollen. Zabludowski meint, dass es unmöglich sei ohne Heilgehilfen auszukommen. Es entspinnt sich nun eine mitunter sehr lebhaft geführte Debatte über die ärztliche und Laienmassage, wobei zwar eine Einigung nicht erzielt wird, aber die Mehrheit doch zu der Ansicht hinzuneigen scheint, dass die letztere, wo sie aus rein materiellen oder lokalen Gründen nicht zu umgehen ist, lediglich unter der ausschliesslichen Aufsicht und Verantwortlichkeit des Arztes erfolgen darf.

Koll. Gunzburg (Antwerpen), welcher zur Vervollständigung dieses Berichtes durch Erlaubnis zur Einsichtnahme in das offizielle Protokoll beitrug, sei hiermit der Dank ausgesprochen.

\title{
IV. Bücher.
}

F. Klaussner. Über Missbildungen der menschlichen Gliedmassen. Neue Folge. Wiesbaden. J. F. Bergmann. 1905. 41 S., 32 Abbildungen. Preis $2 \mathrm{Mk}$.

Verf. teilt aus einer grösseren Anzahl von Beobachtungen eine Reihe interessanter Fälle von Missbildungen der menschlichen Gliedmassen zwecks Fortsetzung und Ergänzung seiner bekannten Publikation, welche im Jahre 1900 über 
das gleiche Thema und im gleichen Verlag erschienen ist, mit. Nach Anuahme des Verf. dürfte der namentlich von $\mathrm{Kü} \mathrm{mmel} \mathrm{vertretene} \mathrm{Standpunkt,} \mathrm{dass} \mathrm{in}$ frühester Entwickelungszeit einwirkende mechanische Momente vielfach die Missstaltungen veranlassen, durch die vorliegenden Befunde wiederum neuerdings Bestätigung finden. Die Fälle betreffen Riesenwuchs, Flughautbildung, Radiusdefekt, Syn- und Polydaktylie, Spalthand, Brachydaktylie, Elektrodaktylie, Missstaltungen des Daumens, der Finger etc.

Die Abbildungen sind sehr instruktiv. Die Ausstattung des Werkes ist eine vorzügliche.

J. Riedinger.

W. Pieper. Betrachtungen über das Heilverfahren bei den Berufs. genossenschaftenundVorschlägezurpraktischen Durchführung desselben. Darmstadt 1904. Verlag von B. L. Schlapp.

Eine Broschüre, die von den Vorständen aller Berufsgenossenschaften, aber auch von jedem Arzte gelesen werden müsste, der in die Lage kommt, Unfallverletzte zu behandeln. Als kurze Inhaltsangabe der Broschüre kann man die Schluss. worte derselben verzeichnen: „Erst Heilverfahren, dann Rente“. P i e per ist Sekretär der land- und forstwirtschaftlichen Berufsgenossenschaft für das Grossherzogtum Hessen, und er glaubt aus seiner langjährigen Tätigkeit in der Unfallversicherungspraxis die Erfahrung gemacht zu haben, dass vielfach ein intensives und ausreichendes Heilverfahren, insbesondere eine angemessene Nachbehandlung von den Berufsgenossenschaften erst dann eingeleitet wird, wenn es zu spät ist. Jeder, der mit den einschlägigen Verhältnisse bekannt ist, insbesondere auch die Leser dieser Zeitschrift, werden die Richtigkeit dieser Beobachtung bestätigen. Das Gesetz hat den Berufungsgenossenschaften das Recht gegeben, die Heilbehandlung schon während der ersten 13 Wochen nach dem Unfall zu übernehmen, was doch offeusichtlich in dem eigensten Interesse der Berufsgenossenschaft selbst liegt, da ja in erster Linie durch ein zweckentsprechendes Heilverfahren die schädigenden Folgen des Unfalls beseitigt werden können. Aber nur wenig machen die Berufsgenossenschaften von diesem Rechte Gebrauch! Gewiss gibt es rühmliche Ausnahmen, und Pieper selbst benennt mebrere Berufsgenosseuschaften, die auf dem Gebiete des Heilverfahrens Hervorragendes leisten. Aber im grossen und ganzen muss man doch sagen, dass die Berufsgenossenschaften erst nach Ablauf der Karenzzeit einzutreten pflegen und vorher die Fürsorge für den Verletzten den Krankenkassen überlassen. Ja, es wird sogar darüber geklagt, dass nach Inkrafttreten der letzten Krankenversicherungsnovelle, nach welcher die Krankenkassen zur Leistung von Krankenunterstützung für 26 Wochen verpflichtet sind, sich bei einzelnen Berufsgenossenschaften die Tendenz zeigt, noch nach Beginn der 14 . Woche das Heilverfahren den Krankenkassen zu überlassen, weil ihnen die Kur auf diese Weise billiger kommt. Mit Recht weist $\mathrm{P}$ i e p e $\mathrm{r}$ auf die Leistungsunfähigkeit der meisten kleinen Krankenkassen hin, die eine Heilbehandlung in den grossen modernen Anstalten in der Regel nicht gewähren können, insbesondere dann nicht, wenn sich eine solche Anstalt nicht in dem Orte befindet, wo die Krankenkasse ihren Sitz hat. Noch viel trauriger liegt die Sache bei den land- und forstwirtschaftlichen Arbeitern, die sich in der Regel einer Krankenkassenfürsorge überhaupt nicht erfreuers.

Mit eindringlichen Worten und unter Anführung mancherlei Beispiele aus der Praxis verlangt nun $\mathrm{Pieper}$, dass die Berufsgenossenschaften möglichst frühzeitig ein möglichst intensives Heilverfahren übernehmen. Er fasst seine Forderungen in folgende Sätze zusammen:

1. In allen geeigneten Fällen muss ein Heilverfahren, welches auf die Wiederberstellung der Funktion abzielt, eingeleitet werden.

2. Dieses Heilverfahren lässt sich zweckmäsig nur durchführen in einer mit allen in Betracht kommenden Heilfaktoren ausgerüsteten Anstalt. 
3. Das Heilverfahren muss möglichst früzeitig in unmittelbarem Anschluss an die anatomische Heilung, $d . h$. in den meisten Fällen schon vor Ablauf der 13 Woche nach Fintritt des Unfalls einsetzen.

4. Auf die Genauigkeit der Gutachten muss weit mebr Wert gelegt werden, wie bisher. Wir müssen eine präzise Diagnose verlangen, eine exakte Funktionsprüfung und eine möglichst genaue und zutreffende Prognose.

5. Die Rentenabschätzung wäre am geeignetsten von der Berufsgenossenschaft selbst vorzunehmen.

6. Es wäre anzustreben, dass den Berufsgenossenschaften die gesetzliche Verpflichtung auferlegt würde, bei allen Fällen, die nach der Art der Verletzung eine Behandlung über die 13 Wochen hinaus voraussehen lassen, schon vor Ablauf der Karenzzeit in das Heilverfahren einzugreifen.

7. Es ist dahin zu wirken, dass den Berufsgenossensahaften bezüglich einer Ermässigung der Eisenbahnpreise für ibre Verletzten die gleichen Vergünstigungen eingeräumt werden, wie sie den Krankenkassen jetzt schon gewährt sind.

Wir können nur hoffen, dass diese Vorschläge in den Kreisen der Berufsgenossenschaft gehört werden. Vermissen tun wir allerdings einen Punkt, nämlich entsprechende Massnahmen, um den aus den Anstalten entlassenen Verletzten eine ibrer verminderten Leistungsfähigkeit entsprechende Arbeit $z \mathfrak{u}$ beschaffen, obwohl es doch heutzutage dem Halbinvaliden so ausserordentlich schwer fällt, passende Arbeit zu finden, ganz besonders in den Städten. Doch das ist ein Kapitel für sich. Seelmann (Königsberg i. Pr.).

Dessauer und Wiesner (A schaffenburg). Kompendium der Röntgenographie. Ein praktisches Handbuch. 415 S. Text mit 201 Illustrationen, 11 Fehlertafeln in Autotypie und 12 radiograph. Tafeln. Iseipzig 1905 bei $\mathrm{O}$ tto $\mathrm{Nemnich}$. Gebd. Mk. 25.-

Das vorliegende Buch will ein Handbuch der Röntgenaufnahme sein, das sich auf wissenschaftlicher Basis unmittelbar in den Dienst der Praxis stellt. Es zerfällt demnach in 3 Teile: in den physikalischen, den photographischen und den praktischen.

Der I. Teil beginnt mit der sehr interessanten Entwickelungsgeschichte der Röntgentechnik. Fr schildert anschaulich die grossen Schwierigkeiten, die zu überwinden waren, bis die gegenwärtige Höhe erreicht werden konnte. Am interessantesten ist zweifellos in diesem Kapitel die Darstellung des Gegensatzes zwischen Hamburger (Walter und Albers-Schönberg) und Aschaffenburger (Verfasser) Richtung. Die erstere erhält dabei einige der Darstellung nach wohlverdiente Abfubren und man darf gespannt sein, was sie zur Verteidigung vorzubringen hat.

Das II. Kapitel behandelt das Wesen der Röntgen- und Bequerel-Strahlen; ihm folgt als III. die elektrischen Teile des Röntgenapparates und ihr Bau. Es ist zweifellos das theoretisch wichtigste Kapitel, da es uns in einer bisher noch nicht dagewesenen Gründlichkeit einführt in die für das Röntgengebiet wichtigen physikalischen Begriffe, in den Bau des Induktoriums, in die Vor- und Nachteile der verschiedenen Unterbrecher und endlich in die Vorgänge in der Röntgenröhre selber. Das Schlusskapitel des I. Teiles ist dem Bau und dem Betriebe der Röntgenstationen gewidmet; in liberalster Weise ist hier den verschiedensten Konkurrenzfirmen Raum zur Beschreibung ihrer Röntgenanlagen gewährt. So sind durch eigene Beschreibung ihrer Chef-Ingenieure vertreten die Polyphos-Gesellschaft in München, Klingelfuss \& Co. in Basel, Nostir \& Koch in Chemnitz, sowie das Elektrotechnische Laboratorium Aschaffenburg.

Der II. Hauptteil des Buches behandelt die photographischen Grundlagen, die Einrichtungen und Apparate für den Negativprozess, die Anleitung zum Entwickeln und zur Korrektur der Negative, sowie das Positiv- und Diapositiv-Verfahren. Ein besonderer Abschnitt ist der Beurteilung der Negative, der so überaụs wichtigen 
Berichte.

Plattenkritik gewidmet, die durch 11 Fehlertafeln in Autotypie erläutert wird. Es bedarf keines besonderen Hinweises, wie ausserordentlich wichtig es für den, der nach fehlerfreien Bildern strebt, ist, hier Ursache und Bedeutung mancher Aufnahme- und Entwickelungsfehler, sowie die Mittel und Wege zu ihrer Vermeidung bezw. Beseitigung an praktischen Beispielen dargestellt zu finden.

Der III. Teil ist der Aufnahmetechnik der einzelnen Körpergegenden gewidmet. Er entbält die praktische Anleitung zu jeder dem Arzte vorkommenden Röntgen-Aufnahme. Für eine jede ist die Lagerung des Patienten abgebildet, sowie die Röhrenwah], die Aufnahmerichtung und die durchschnittliche Expositionszeit angegeben. Besonders ausführlich, weil besonders schwierig, sind die Aufnahmen des Kopfes, des Thorax und des Abdomens geschildert; daneben sind selbstverständlich auch die Extremitäten-Aufnahmen, die ja das bei weitem grösste und praktisch wichtigste Kontingent aller Röntgenbilder darstellen, zu ihrem vollen Rechte gekommen. Neben den Lagerungsbildern werden die anatomischen Verhältnisse durch kurze Skizzen veranschaulicht, während 12 Tafeln mit meist sehr schönen Röntgenphotographien die gewonnenen Resultate illustrieren und damit zugleich einen Beweis für die grosse Leistungsfähigkeit der Aschaffenburger Richtung bringen. Im letzten Kapitel ist die Stereoskopie im Röntgenverfahren ein. gehend dargestellt.

Hiermit wäre ein kurzer Überblick über den Inbalt des vorliegenden Werkes gegeben. Praktischer würde es wohl sein, wenn der III. Teil vor dem II. seine Stelle fände, denn er ist die unmittelbare Anwendung des im I. Teile Abgehandelten, auf das er wiederholt zurückgreift; auch rein äusserlich genommen, kommt ja stets die Aufnahme zuerst, dann das Entwickeln. Ferner wäre es zu begrüssen, wenn in einer Neuauflage ein Kapitel auch der Orthodiagraphie gewidmet würde, da die mit derselben angefertigten Skizzen uns auch ins Gebiet der Röntgenographie zu gehören scheinen. Doch dies nur nebenbei. Alles in allem stellt zweifellos das vorliegende Buch das vollkommenste und ausführlichste unter allen uns bekannten Lehrbüchern des Röntgenverfahrens dar. Dabei ist in der Ausstattung nichts gespart, die Abbildungen sind reichlich und meist vortrefflich, so dass sich das Werk sicher in kurzer Zeit einen grossen Freundeskreis erwerben wird. W. Becker (Bremen).

Grashey. Atlas typischer Röntgenbilder vom normalen Menschen. Lehmanns Med. Atlanten. Neue Folge. Bd. V. München 1905. Preis in Leinen gebd. M. $16 .-$

Vorliegender Atlas will denjenigen, der im Lesen von Röntgenbildern unerfahren ist, in das Studium derselben einführen. Denn wer im Röntgenogramm pathologische Befunde aufdecken will, muss zuerst das Normalbild genau im Kopfe haben. Wie kompliziert und schwer zu deuten aber dasselbe oft sein kann, weiss jeder, der sich mit diem Gebiete beschäftigt. Um so dankbarer wird er einem Führer sein, der nach Form und Inbalt so gelungen ist, wie der vorliegende.

Dem Atlas vorangestellt ist eine kurze physikalisch-technische Abhandlung, welche zur Orientierung über die Aufnahme und zur Deutung des Schattenbildes, namentlich seiner Projektionsverbältnisse, sehr zweckdienlich ist. Bei den Abbildungen selbst steht der Text immer auf der gegenüberliegenden Seite, so dass man Bild und Erklärung gleichzeitig vor Augen hat; diese Anordnung untersebeidet sich in sehr vorteilhafter Weise von derjenigon der Hamburger Atlanten. Der Bildertext beginnt jedesmal mit der Art der Aufnahme und bespricht, wo es nötig erscheint, ausführlich die Aufnahmetechnik, die mancよe schätzenswerten Winke enthält, um Fehler der Projektion etc. zu vermeiden. Die Erklärung selbst nimmt auf Zahlen Bezug, die im Bilde eingedruckt sind; auch diese Anordnung ist äusserst praktisch, denn sie gestattet eine schnelle Orientierung, ohne die Klarheit des Bildes zu stören. Wichtig ist, dass stets der Punkt, auf dem die Röhre eingestellt war, 
durch ein Kreuz markiert ist, wodurch die richtige Vorstellung der Projektion ermöglicht wird. Manche Bilder sind besonders erläutert durch beigegebene schematische Zeichnungen; bei einigen Bildern ist auch die Knochenkontur direkt eingetragen.

Im ganzen enthält der Atlas 97 Autotypien, sowie 42 Konturzeichnungen. Die chirurgisch wichtigen Teile des Skeletts sind dabei mit ziemlich gleicher Gründlichkeit behandelt: der Kopf mit seinen Höblen, die wichtigten Teile der Wirbelsäule mit dem Schultergürtel, Schulter, Elibogen und Handgelenk, sowie Hüfte, Knie und Fuss.

Die Aufnahmen sind in den verschiedensten Richtungen angefertigt, so dass wohl jeder vorkommende Fall ein Analogon finden dürfte. Die verschiedenen Altersstufen sind zwar nicht systematisch durchgeführt, das würde auch im Rahmen eines A tlanten gar nicht möglich sein, doch sind bei allen grossen Gelenken Bilder von Erwachsenen und von Kindern vertreten. Auch die praktisch wichtigen Varietäten des Skeletts sind überall da, wo sie zur Verwechselung mit pathologischen Zuständen Veranlassung geben könnten, gebührend berücksichtigt.

Die Bilder sind durchweg schön und klar ausgeführt; manchen sieht man die Sublimatverstärkung (oder Retouche?) ein wenig an, doch hat die Deutlichkeit für den praktischen Gebrauch dadurch nur gewonnen. Mit Freuden ist es zu begrüssen, dass auf die Bilder aus der inneren Medizin mit ihren mehr zweifelhaften praktischen Ergebnissen fast ganz verzichtet wurde.

Die äussere Ausstattung des Buches ist eine ganz vorzügliche, das Format ist sehr handlich, der Preis im Hinblick auf die grosse Summe des Gebotenen ein sehr mässiger, so dass der Atlas zweifellos die weiteste Verbreitung finden wird, die er nach Form und Inhalt verdient. W. Becker (Bremen).

Hermann Lossen (Darmstadt). Die Ernst-Ludwig-Heilaustalt. Beiträge zur Anwendung der Physikalischen Heilmethoden. Darmstadt, Schlapp. 1905. 492 Seiten.

Verf. bietet uns hier in Gestalt einer Anstaltsbeschreibung ein förmliches, durchaus wissenschaftlich nach dem neuesten Stand der Literatur bearbeitetes Lehrbuch. Der Leser findet sowohl eine erschöpfende, theoretische Darstellung als auch eine gute Anleitung der praktischen Durchführung der einzelnen Methoden. Verf. schöpft aus den Erfahrungen, die er in seiner mustergültigen physikalisch. therapeutischen Heilanstalt in Darmstadt erworben hat.

Der Inhalt teilt sich in folgende Kapitel: Einleitung; Bewegungs- und Übungstherapie, Massage; Thermotherapie; Radiotherapie und Phototherapie; Inhalations- und Pneumatotherapie; Hydrotheropie; Nachbehandlung Unfallverletzter.

J. Riedinger.

\section{Journalliteratur.}

a) Referent: Privatdozent Dr. Arnd in Bern.

Spitzy. Die Bedeutung der Nervenplastik für die Orthopädie. (Zschrft. f. orth. Chirurgie. Bd. 13.)

$\mathrm{S}$. bespricht ausführiich die gegenwärtige physiologische Basis der Lehre von der Regeneration der Nerven. Eingehende Untersuchung der am Menschen gewonnenen Erfahrungen. Er empfiehlt die seitliche Implantation, die ihm bei den Hunden sehr gute Resultate gegeben hat. Beim Menschen sind ja bis jetzt die Resultate nicht so glänzende. Einen benachbarten Nerven sollte man nur dann ganz zum $Z$ wecke der Plastik ausschalten, wenn er in seiner Funktion wirklich unbedeutend ist und die Funktion des zu retlenden sehr wichtig. Bei der Naht 